\title{
Risk factors, clinical features and outcome of new-onset acute kidney injury among critically ill patients: a database analysis based on prospective cohort study
}

Yi-Jia Jiang ${ }^{1}$, Xiu-Ming Xi' ${ }^{2}$, Hui-Miao Jia ${ }^{1}$, Xi Zheng ${ }^{1}$, Mei-Ping Wang ${ }^{3}$, Wen $\mathrm{Li}^{2}$ and Wen-Xiong $\mathrm{Li}^{1 *}$

\begin{abstract}
Background: Acute kidney injury (AKI) newly-emerged in intensive care unit (ICU), has not been thoroughly studied in previous researches, is likely to differ from AKI developed before ICU admission. This study aimed to evaluate the incidence, risk factors, clinical features and outcome of new-onset AKI in critically ill patients.

Methods: The data of present study derived from a multicenter, prospective cohort study in17 Chinese ICUs (January 2014 - August 2015). The incidence, risk factors, clinical features and survival analysis of new-onset AKI were assessed.

Results: A total of 3374 adult critically ill patients were eligible. The incidence of new-onset AKI was $30.0 \%$ ( $n=$ 1012). Factors associated with a higher risk of new-onset AKI included coronary heart disease, hypertension, chronic liver disease, use of nephrotoxic drugs, sepsis, SOFA score, APACHEll score and use of vasopressors. The new-onset AKI was an independent risk factor for 28-day mortality (adjusted hazard ratio, 1.643; 95 \% Cl, 1.370-1.948; $P<0.001$ ). 220 (21.7\%) patients received renal replacement therapy (RRT), 71 (32.3\%) of them were successfully weaning from RRT. More than half of the new-onset AKI were transient AKI (renal recovery within $48 \mathrm{~h}$ ). There was no statistical relationship between transient $\mathrm{AKI}$ and 28-day mortality (hazard ratio, 1.406; $95 \% \mathrm{Cl}, 0.840-1.304 ; P=0.686$ ), while persistent AKI (non-renal recovery within $48 \mathrm{~h}$ ) was strongly associated with 28-day mortality (adjusted hazard ratio, 1.486; $95 \% \mathrm{Cl}, 1.137-1.943 ; P<0.001)$.
\end{abstract}

Conclusions: New-onset AKI is common in ICU patients and is associated with significantly higher 28-day mortality. Only persistent AKI, but not transient AKI is associated with significantly higher 28-day mortality.

Keywords: Acute kidney injury, New-onset, Incidence, Risk factors, Outcome

\footnotetext{
* Correspondence: liwx1126@163.com

'Department of Surgical Intensive Critical Unit, Beijing Chao-yang Hospital, Capital Medical University, 8 Gongren Tiyuchang Nanlu, Chaoyang District, 100020 Beijing, China

Full list of author information is available at the end of the article
}

(C) The Author(s). 2021 Open Access This article is licensed under a Creative Commons Attribution 4.0 International License, which permits use, sharing, adaptation, distribution and reproduction in any medium or format, as long as you give appropriate credit to the original author(s) and the source, provide a link to the Creative Commons licence, and indicate if changes were made. The images or other third party material in this article are included in the article's Creative Commons licence, unless indicated otherwise in a credit line to the material. If material is not included in the article's Creative Commons licence and your intended use is not permitted by statutory regulation or exceeds the permitted use, you will need to obtain permission directly from the copyright holder. To view a copy of this licence, visit http://creativecommons.org/licenses/by/4.0/ The Creative Commons Public Domain Dedication waiver (http://creativecommons.org/publicdomain/zero/1.0/) applies to the data made available in this article, unless otherwise stated in a credit line to the data. 


\section{Background}

Acute kidney injury (AKI) occurs in more than $50 \%$ of intensive care patients and is associated with increased risks of in-hospital mortality and long-term chronic kidney disease [1-3]. AKI caused by diverse exposures and susceptible factors may present different incidence and prognosis [4]. Most AKI appears in the first $72 \mathrm{~h}$ after patients are transferred to intensive care unit (ICU) [57], but some critically ill patients may already have kidney damage before admission to ICU. Patients who developed AKI before ICU are likely to differ from those who develop AKI later on the following days. The appearance time of kidney injury implies the difference in causes and severity of the disease, which results in different clinical manifestations and outcomes. However, few studies have defined the exact time when AKI appears.

The severity of AKI is usually graded by the classifications such as the risk, injury, failure, loss of kidney function, and end-stage renal disease (RIFLE), acute kidney injury network (AKIN) and kidney disease improving global outcomes (KDIGO) staging system. More severe AKI is associated with poorer outcomes. However, these classification systems all evaluate the magnitude of serum creatinine increase and/or decrease in urine output, without considering factors such as underlying diseases and duration of AKI. Lately, the duration of kidney injury has been identified as an additional important dimension of AKI severity [8-13]. Transient AKI, typically defined as the duration of kidney injury less than 48$72 \mathrm{~h}$, has been recognized with a lower risk of long-term adverse outcomes compare to AKI of longer duration [9-13], while its impact on short-term outcomes remains controversial in different clinical settings. Therefore, to evaluate the clinical characteristics and prognosis of new-onset AKI according to the duration of renal injury in ICU, we performed a retrospective analysis based on a database of a large, prospective cohort study about sepsis epidemiology in critically ill adults from 17 ICUs of 16 regional central hospitals across China which encompassed a wide range of clinical settings and critical care populations.

\section{Methods}

\section{Study setting and population}

The retrospective analysis based on database of a prospective cohort study about sepsis epidemiology sponsored by China Critical Care Sepsis Trial (CCCST) workgroup, which was performed in 17 Chinese ICUs between January 1st, 2014 and August 31st, 2015. A complete list of trial sites is provided in the Supplementary File. The protocol of the study was registered on August 3rd, 2013. The study was approved in all participating ICUs by their Hospital Human Ethics Committee. The chief ethics number was 2013FXHEC-KY2018. The registration number was ChiCTR-ECH-13,003,934. The study followed the ethical principles of the Declaration of Helsinki 1964. Informed consent from patients or their next of kin was obtained before patients joined in the study. All enrolled patients adhere to the following management principles: active treatment of primary disease and complications, and the same principles of treatment with antibiotics, nutritional metabolism and organ support.

We screened patients from the database of $4910 \mathrm{pa}-$ tients. Adult patients were eligible for inclusion if they stayed longer than $48 \mathrm{~h}$ in ICU, and none of the following exclusion criteria was fulfilled: (1) chronic kidney disease (CKD); (2) acquired insufficient data; (3) operated with nephrectomy or kidney transplantation; (4) developed AKI before ICU; (5) renal replacement therapy (RRT) for non-renal indications.

\section{Definitions and clinical endpoints}

The definitions of AKI and AKI classification were depended on the serum creatinine and urine output criteria proposed by Kidney Disease: Improving Global Outcomes (KDIGO) [14]. New-onset AKI was defined as acute kidney injury occurred within 72hours after ICU admission. If a patient could be diagnosed with AKI at the time of admission (elevated serum creatinine or oliguria within the first 6 hours after admission that met the KDIGO criteria), it is considered that AKI was already occurred before ICU admission, and this patient couldn't be diagnosed as new-onset AKI.CKD was defined according to the definition of National Kidney Foundation as estimated glomerular filtration rate (eGFR) $<60 \mathrm{ml} / \mathrm{min} / 1.73 \mathrm{~m}^{2}$ for at least 3 months irrespective of the cause. GFR was estimated with the Cockcroft-Gault formula $[15,16]$. The baseline creatinine was defined as follows: if at least five values were available the median of all values available from 6 months to 6 days prior to enrollment was used. Otherwise, the lowest value in the 5 days prior to enrollment was used. If no pre-enrollment creatinine was available or the emergency patient's serum creatinine was abnormal at the time of admission, the baseline creatinine was estimated using the Modification of Diet in Renal Disease (MDRD) equation assuming that baseline eGFR is $75 \mathrm{ml} / \mathrm{min}$ per $1.73 \mathrm{~m}^{2}$ [17]. The diagnosis of sepsis and septic shock was according to the sepsis 3.0 definition of the American College of Chest Physicians/ Society of Critical Care Medicine criteria [18]. Transient AKI was defined as kidney injury recovered within $48 \mathrm{~h}$ [19]. Persistent AKI was defined as acute kidney injury more than $48 \mathrm{~h}$. Recovery of AKI was defined as the absence of any stage of AKI by either serum creatinine or urine output criteria. For example, a patient with stage 2 AKI would have to have a decrease in serum creatinine to 
less than $150 \%$ of baseline, and be free of periods of oliguria longer than $6 \mathrm{~h}$ [20]. When $48 \mathrm{~h}$ follow-up was not possible due to death or discharge from ICU, AKI was defined as persistent if patients met KDIGO criteria for their last measurement, otherwise as transient. Weaning of RRT was defined as the cessation of RRT within 28 days, and requiring no further support for at least 7 days. Major surgery was defined as the surgery classification of grade 3 or 4 identified by the National Health Commission of China.

The primary endpoint was 28-day mortality. Secondary endpoints were weaning of RRT, length of ICU stay and hospital stay, hospital mortality.

\section{Data collection}

Data were prospectively recorded during the hospital stay. The information collected included demographic characteristics, chronic illnesses, diagnosis, pre-ICU medications (whether or not used nephrotoxic drugs, nephrotoxic drugs included angiotensin converting enzyme inhibitors, non-steroidal anti-inflammatory drug, amikacin and amphotericin B) and treatment, the reason for ICU admission, whether or not infection, acute physiology and chronic health evaluation (APACHE II) (based on data recorded during the first $24 \mathrm{~h}$ of ICU admission), baseline serum creatinine, creatinine values every $12 \mathrm{~h}$ and hourly urine output on ICU admission and thereafter until transferred out of ICU, days of mechanical ventilation, use of vasoactive drugs, sequential organ failure assessment (SOFA) score and the value of lactate every day in the first 3 days after ICU admission. We also collected time of diagnosing AKI, initiation and weaning of RRT, ICU stay, hospital stay, hospital mortality and 28-day mortality.

\section{Statistical analysis}

Continuous variables were presented as mean \pm standard deviation (SD) or median values (25th and 75th percentiles), categorical variables were presented as percentiles. Continuous data between two groups was compared using the Student's t-test or Mann-Whitney U tests, and categorical variables used the Chi-square test or Fisher's exact test. To assess the association of variables and new-onset AKI within the entire cohort, a logistic regression analysis was conducted to calculate the odds ratios (ORs) and $95 \%$ confidence intervals (CIs). Regression models used a backward procedure with a significance level of 0.1. The 28-day survival rate was calculated using the Kaplan-Meier method. The survival comparison among the groups was performed using the log-rank test. Cox proportional hazards model was used to graphically describe the in-hospital survival. The hazard ratios (HRs) and $95 \%$ confidence intervals for mortality rates were calculated using the Cox proportional hazard model after adjustment for potential confounders. For all analyses, statistical significance was indicated by two-sided $P<0.05$. SPSS statistics 24 (IBM, Chicago, IL) and R 3.6.1 (R Project for Statistical Computing) were used for statistical analyses.

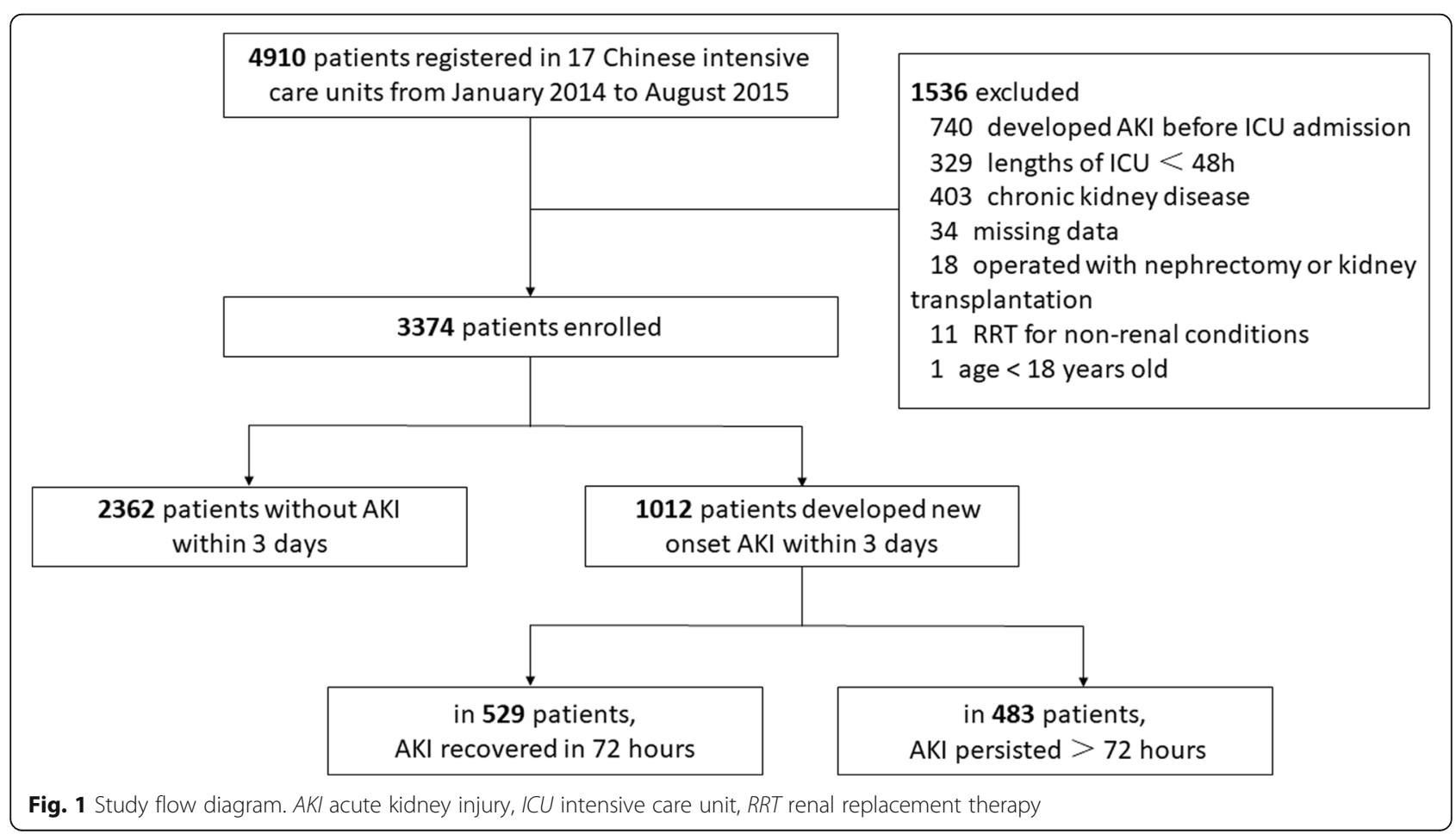




\section{Results}

\section{Demographics}

We identified 3374 patients from 17 ICUs after applying all inclusion and exclusion criteria (Fig. 1). The median [interquartile range (IQR)] age of the patients was 64 (49-76) years. Of these, all were self-identified as Asian and $64 \%$ were male. Patients mainly came from operation room $(34.4 \%, n=1162)$, other department $(31.7 \%$, $n=1071)$, emergency room $(21.0 \%, n=709)$ and others $(12.8 \%, n=432)$. The median APACHE II score and SOFA score was 16 and 5 within $24 \mathrm{~h}$ after ICU admission, respectively. Sepsis was diagnosed at admission for 1183 (35.1\%) patients. 1912 (56.7\%) patients received mechanical ventilation. The median length of stay in hospital was 18 (11-27) days. The unadjusted inhospital mortality rate was $20.5 \%(n=691)$. Table 1 listed all the baseline characteristics and covariates included in the regression models for the outcome of newonset AKI and 28-day mortality.
Incidence of new-onset AKI in different clinical settings 1174 (34.8\%) developed AKI in the first 7 days in ICU, among them, new-onset AKI (AKI occurred within $72 \mathrm{~h}$ after ICU admission) developed in 1012 (30.0\%) patients. $586(57.9 \%)$ of the new-onset AKI developed kidney injury on the first day of ICU. The number of new-onset AKI developed per day was shown in Fig. 2. The median SOFA score on the day of AKI diagnosis was 7 (4-10). Half of new-onset AKI patients had sepsis before the diagnosis of AKI, 286 (56.5\%) of them were septic shock. AKI severity according to the KDIGO classification was determined to be stage 1 in 464 (45.8\%) patients, stage 2 in 177 (17.5\%) patients, and stage 3 in $371(36.7 \%)$ patients. Among the new-onset AKI patients, $220(21.7 \%)$ patients received RRT. The most common causes of RRT were oliguria/anuria ( $n=179$, $81.4 \%)$, followed by severe acidosis $(\mathrm{pH}<7.15)(n=79$, $35.9 \%)$, refractory hyperkalemia $\left(\mathrm{K}^{+}>6 \mathrm{mEq} / \mathrm{L}\right)(n=64$, $29.1 \%)$ and fluid overload with organ edema $(n=20$,

Table 1 Baseline characteristics of patients stratified by new-onset acute kidney injury

\begin{tabular}{|c|c|c|c|c|}
\hline \multirow[t]{2}{*}{ Variables } & \multirow{2}{*}{$\begin{array}{l}\text { All patients } \\
N=3374\end{array}$} & \multirow{2}{*}{$\begin{array}{l}\text { No AKI } \\
N=2362\end{array}$} & \multirow{2}{*}{$\begin{array}{l}\text { New onset AKI } \\
N=1012\end{array}$} & \multirow[t]{2}{*}{$P$ value } \\
\hline & & & & \\
\hline Male $n(\%)$ & $2144(63.5)$ & $1500(63.5)$ & $644(63.6)$ & 0.942 \\
\hline Age (years) & $64(49-76)$ & $62(48-75)$ & $67(52-79)$ & $<0.001$ \\
\hline $\mathrm{BMI}\left(\mathrm{kg} / \mathrm{m}^{2}\right)$ & $22.6(20.0-24.5)$ & $22.5(19.8-24.5)$ & $22.8(20.3-24.5)$ & 0.136 \\
\hline \multicolumn{5}{|l|}{ Chronic comorbidities } \\
\hline COPD/asthma $n(\%)$ & $255(7.6)$ & $185(7.8)$ & $70(6.9)$ & 0.357 \\
\hline Coronary heart disease $n(\%)$ & $542(16.1)$ & $342(14.5)$ & $200(19.8)$ & $<0.001$ \\
\hline Hypertension $n(\%)$ & $1054(31.2)$ & $663(28.1)$ & $391(38.6)$ & $<0.001$ \\
\hline Diabetes $n(\%)$ & $522(15.5)$ & $334(14.1)$ & $188(18.6)$ & 0.001 \\
\hline Cancer $n(\%)$ & $369(10.9)$ & $251(10.6)$ & $118(11.7)$ & 0.378 \\
\hline Chronic liver disease $n(\%)$ & $91(2.7)$ & $52(2.2)$ & $39(3.9)$ & 0.007 \\
\hline APACHEII & $16(10-22)$ & $15(9-20)$ & $18(13-25)$ & $<0.001$ \\
\hline SOFA & $5(3-8)$ & $4(3-7)$ & $7(4-10)$ & $<0.001$ \\
\hline Sepsis $n(\%)$ & $1183(35.1)$ & $677(28.7)$ & $506(50.0)$ & $<0.001$ \\
\hline Mechanical ventilation $n(\%)$ & $1912(56.7)$ & $1330(56.3)$ & $582(57.5)$ & 0.519 \\
\hline Use of vasopressors $n(\%)$ & $746(22.1)$ & $411(17.4)$ & $335(33.1)$ & $<0.001$ \\
\hline Baseline creatinine $(\mu \mathrm{mol} / \mathrm{L})$ & $83(66-93)$ & $82(66-93)$ & $84(65-97)$ & 0.268 \\
\hline Use of nephrotoxic drugs $n(\%)$ & $362(10.7)$ & $195(8.3)$ & $167(16.5)$ & $<0.001$ \\
\hline LOS in ICU (days) & $6(3-13)$ & $6(3-12)$ & $7(4-15)$ & $<0.001$ \\
\hline LOS in hospital (days) & $18(11-27)$ & $18(11-27)$ & $18(10-27)$ & 0.377 \\
\hline \multicolumn{5}{|l|}{ Mortality } \\
\hline ICU mortality & $535(15.9)$ & $245(10.4)$ & $290(28.7)$ & $<0.001$ \\
\hline 28-day mortality & $573(17.0)$ & $276(11.7)$ & $297(29.3)$ & $<0.001$ \\
\hline Hospital mortality & $691(20.5)$ & $346(14.6)$ & $345(34.1)$ & $<0.001$ \\
\hline
\end{tabular}

Continuous variables are presented as median and interquartile range $B M I$ body mass index, COPD chronic obstructive pulmonary disease, APACHEIl acute physiologic and chronic health evaluationll, SOFA sequential organ failure assessment, LOS length of stay 


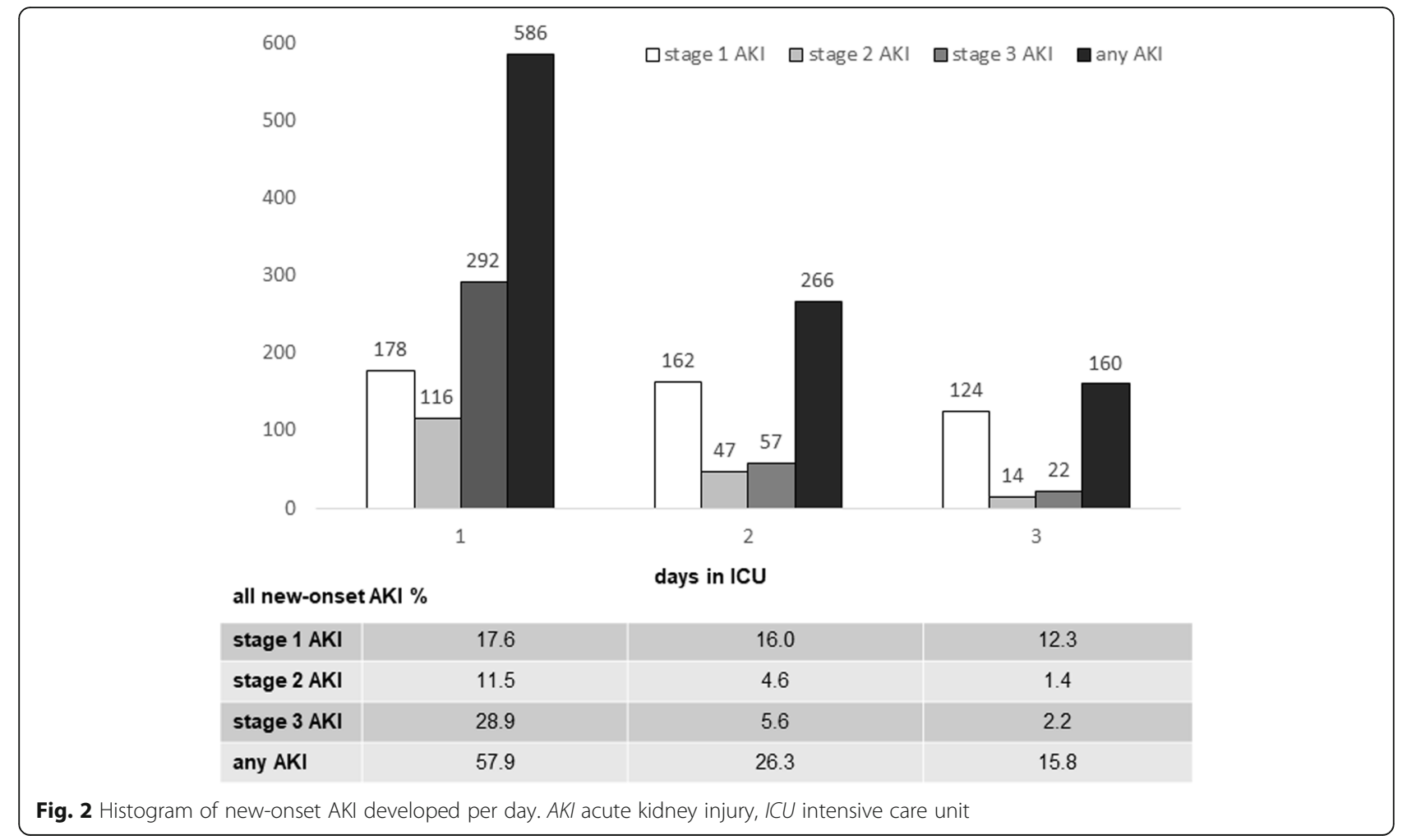

$9.1 \%) .32 .3 \%(n=71)$ of them were successfully weaning from RRT.

The incidence of new-onset AKI varied among patients transferred to ICU for different reasons (Fig. 3). The three leading reasons with the highest incidence of new-onset AKI were sepsis (42.8\%), major surgery $(32.1 \%)$ and cardiac disease $(25.5 \%)$. Patients transferred to ICU due to sepsis had the highest incidence of newonset AKI. Among them, stage 3 AKI accounted for $21.4 \%$ of new-onset AKI, which is also the highest proportion among different populations.

\section{Risk factors for development of new-onset AKI}

For all demographic data, clinical presentation data and laboratory findings presented in Table 1 , we initially evaluated, using univariate analysis, each variable that displayed a statistically significant difference $(p<0.05)$ between new-onset AKI and non-AKI. On the univariate analysis, the risk factors associated with development of new-onset AKI were age, coronary heart disease, diabetes, hypertension, chronic liver disease, use of nephrotoxic drugs, sepsis, SOFA score, APACHEII score and use of vasopressors at ICU admission (Table 2). The above 10 variables were further processed using a multivariable logistic regression model, which selected eight variables that were predictive of new-onset AKI, including coronary heart disease, hypertension and chronic liver disease, use of nephrotoxic drugs, sepsis, SOFA score, APACHEII score and use of vasopressors.

New-onset AKI as an independent risk factor for death $573(17.0 \%)$ of enrolled patients had died within 28 days. Non-survivors were more likely to develop new-onset AKI than survivors. The 28-day mortality rate of the patients with new-onset AKI was higher than that of the non-AKI patients $(P<0.001)$. Patients were divided into two groups (new-onset AKI and non-AKI) for KaplanMeier survival curve analysis. The new-onset AKI group had a significantly lower 28-day survival rate than nonAKI groups $(P<0.001$, Fig. 4 a). In our multivariate cox regression model (Fig. 5), after adjusting for age, comorbidities (COPD/asthma, hypertension, diabetes and cancer), APACHEII, SOFA score, the need for mechanical ventilation, the presence of sepsis and use of vasopressors, new-onset AKI (adjusted hazard ratio, 1.643; $95 \%$ CI, 1.370-1.948; $P<0.001)$ was associated with a higher risk of 28-day mortality.

\section{Stratified analysis by duration of new-onset AKI}

Of the new-onset AKI patients, 529 (52.7\%) patients with AKI recovered within $48 \mathrm{~h}$, namely transient AKI, another $483(47.3 \%)$ patients were persistent AKI (AKI persisted more than $48 \mathrm{~h}$ ). Baseline characteristics stratified by AKI duration were shown in Table S1. Patients 


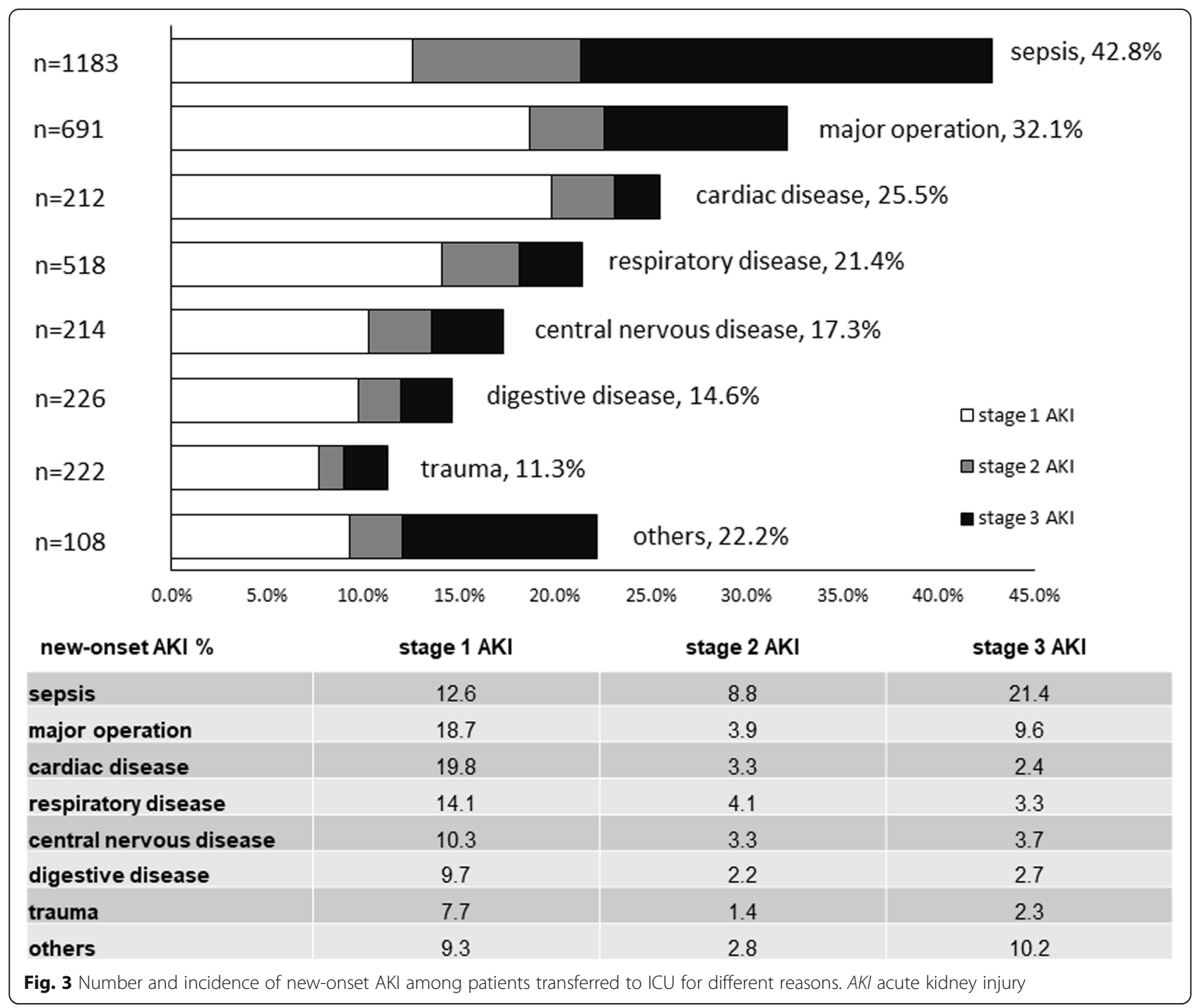

Table 2 Risk factors for new-onset AKI

\begin{tabular}{llcc}
\hline Variables & Crude OR (95 \% Cl) & Adjusted OR (95\% Cl) & $P$ value \\
\hline Coronary heart disease & $1.455(1.200-1.764)$ & $1.319(1.053-1.652)$ & 0.016 \\
Chronic liver disease & $1.781(1.168-2.715)$ & $1.641(1.040-2.588)$ & 0.033 \\
Hypertension & $1.613(1.382-1.884)$ & $1.312(1.085-1.586)$ & 0.005 \\
Sepsis & $2.489(2.138-2.898)$ & $1.636(1.377-1.944)$ & 0.000 \\
SOFA & $1.176(1.151-1.202)$ & $1.096(1.063-1.130)$ & 0.000 \\
APACHE II & $1.071(1.060-1.081)$ & $1.032(1.020-1.045)$ & 0.000 \\
Use of nephrotoxic drugs & $2.196(1.760-2.741)$ & $1.861(1.461-2.370)$ & 0.000 \\
Use of vasopressors & $2.349(1.984-2.781)$ & $1.268(1.025-1.569)$ & 0.029 \\
Age & $1.013(1.009-1.018)$ & $1.003(0.998-1.008)$ & 0.268 \\
Diabetes & $1.385(1.139-1.686)$ & $1.014(0.812-1.266)$ & 0.901 \\
\hline
\end{tabular}



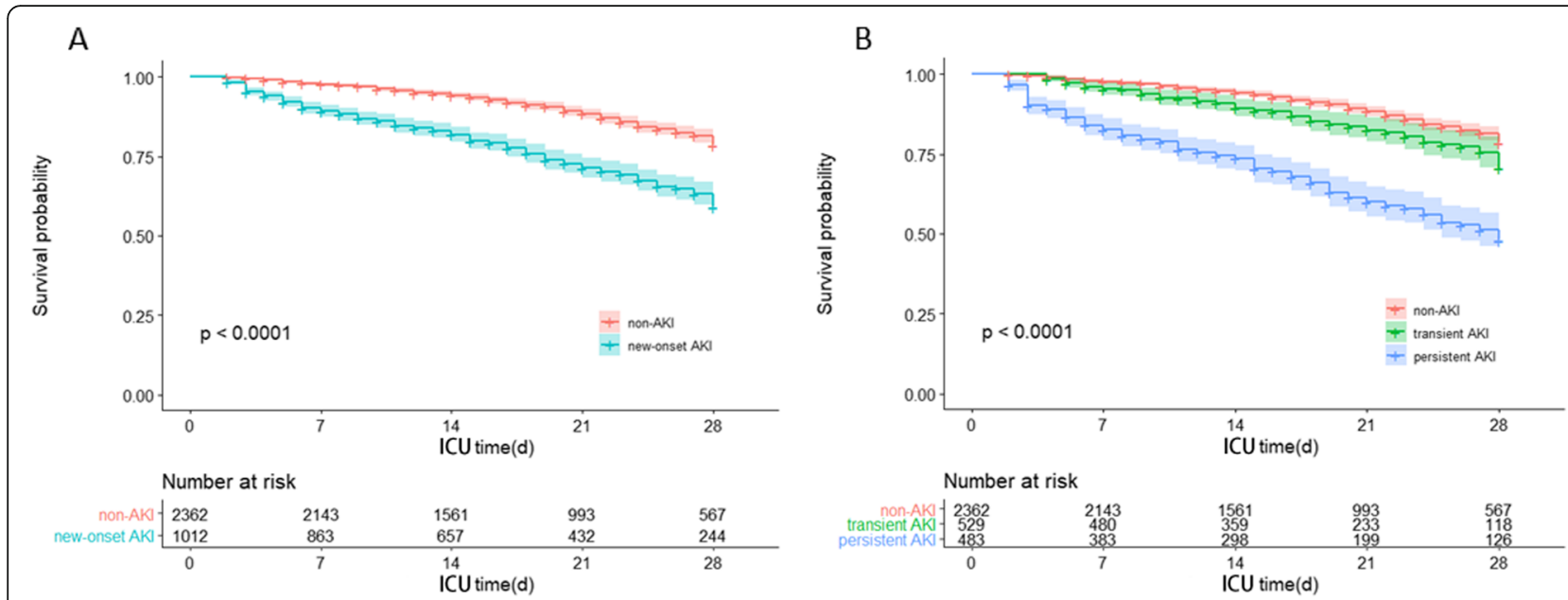

Fig. 4 a Kaplan-Meier survival analysis comparing new-onset AKI and non-AKI groups. b Kaplan-Meier survival analysis comparing non-AKl, transient AKI and persistent AKI groups. $P<0.001$ for all comparisons. Numbers of patients at risk at each time point shown below the graph. AKI acute kidney injury, ICU intensive care unit

who experienced persistent AKI were older and sicker, with higher APACHE II and SOFA scores, and were more likely to have sepsis and vasopressors needs. Table 2 presents outcomes stratified according to AKI duration. After adjustment for confounding covariates, we found that hypertension, baseline creatinine, APACHEII score, SOFA score, sepsis and use of vasopressors were associated with persistent AKI (Table 3). Compared to patients with transient AKI, patients with persistent AKI showed higher 28-day mortality, longer

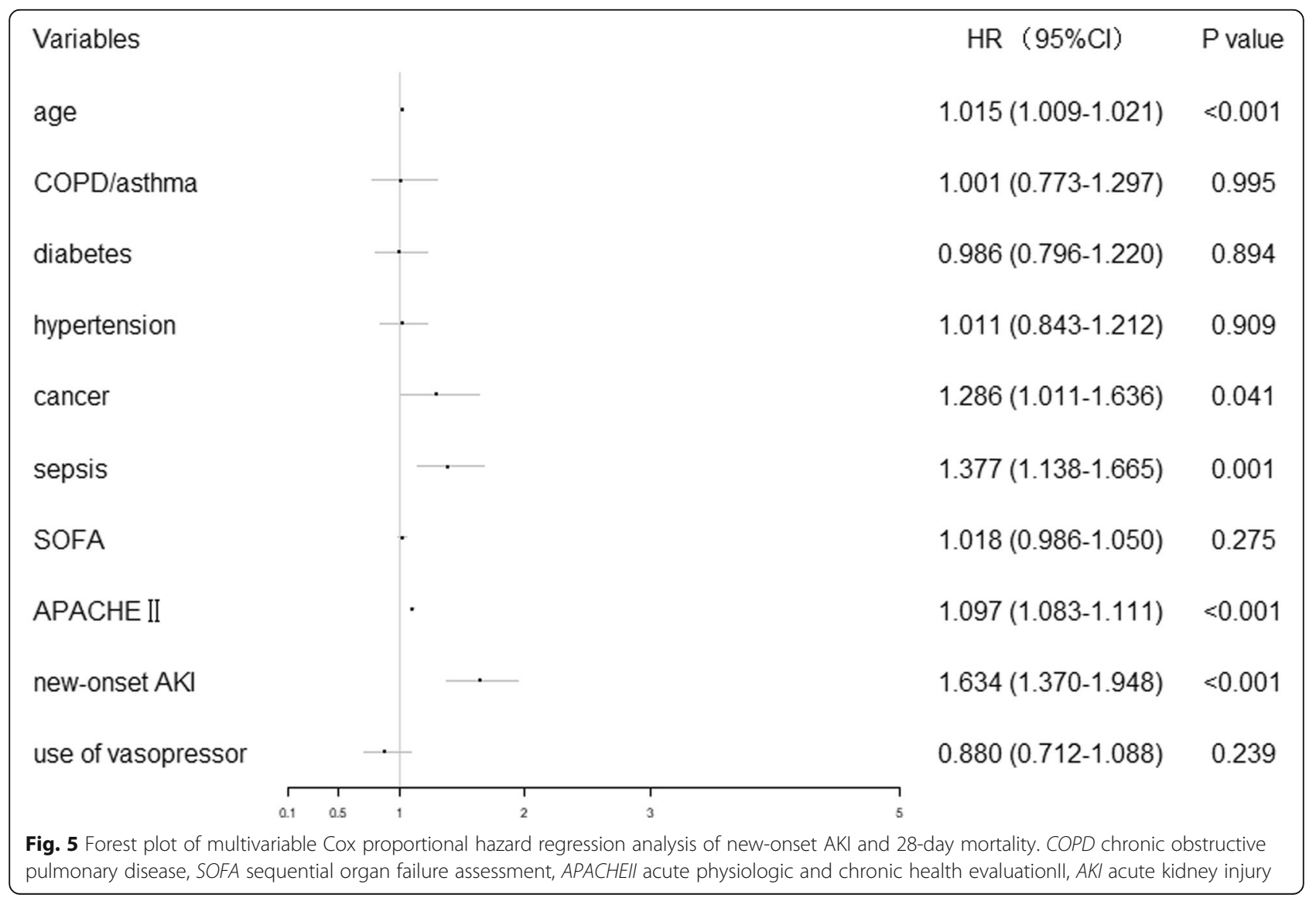


Table 3 Risk factors of persistent AKI

\begin{tabular}{lccc}
\hline Variables & Crude OR $\mathbf{( 9 5} \% \mathbf{C l})$ & Adjusted OR $\mathbf{9 5} \% \mathbf{C l})$ & $\mathbf{P}$ value \\
\hline Hypertension & $1.406(1.091-1.813)$ & $1.412(1.061-1.880)$ & 0.018 \\
Baseline creatinine $(\mu \mathrm{mol} / \mathrm{L})$ & $1.005(1.003-1.007)$ & $1.002(0.099-1.004)$ & 0.163 \\
APACHEII & $1.094(1.075-1.114)$ & $1.057(1.035-1.078)$ & 0.000 \\
SOFA at diagnostic day of AKI & $1.210(1.168-1.253)$ & $1.117(1.068-1.169)$ & 0.000 \\
Sepsis before diagnosis of AKI & $3.784(2.905-4.928)$ & $2.579(1.935-3.439)$ & 0.000 \\
Mechanical ventilaton & $0.920(0.685-1.236)$ & & $0.863(0.626-1.191)$ \\
Use of vasopressors & $1.540(1.184-2.005)$ & 0.371 \\
\hline
\end{tabular}

AKI acute kidney injury, OR odds ratio, APACHEII acute physiologic and chronic health evaluationll, SOFA sequential organ failure assessment

length of ICU and hospital stay (Table 4). Kaplan-Meier curve revealed significantly higher mortality rates in persistent AKI group compared to patients with transient AKI $(P<0.001$; Fig. 4 b) . Survival probabilities at the 28th day were $88.3 \%$ for patients without AKI, $82.0 \%$ for those with transient AKI, and $58.2 \%$ for those with persistent AKI. However, univariate cox proportional hazard model revealed that transient AKI was not associated with 28-day mortality (crude hazard ratio, 1.046, $95 \% \mathrm{CI}$; $0.840-1.304, P=0.686)$. For patients with new-onset AKI, univariate analysis revealed that persistent AKI was independently associated with higher mortality (crude hazard ratio, 2.483; $95 \%$ CI, 1.945-3.169, $P<0.001)$. After adjustment for confounders, the association between persistent AKI and mortality was found to be qualitatively preserved (adjusted hazard ratio, $1.486 ; 95 \%$ CI, $1.137-1.943 ; P<0.001)$.

\section{Center variability}

We observed intercenter variability in the rate of newonset AKI [median, 27.4\%; IQR (18.0 - 36.5\%)], which showed the median rate was similar to the overall rate of new-onset AKI (30.0\%) and the $95 \%$ confidence intervals overlap for the vast majority of sites, suggested the variability of each center did not cause a statistical difference. The rates for each individual center are shown in Table S2 in the Supplementary Appendix.

Table 4 Characteristics of new-onset AKI patients stratified by duration of acute kidney injury

\begin{tabular}{|c|c|c|c|}
\hline & $\begin{array}{l}\text { All new-onset AKI } \\
N=1012\end{array}$ & $\begin{array}{l}\text { Transient AKI } \\
N=529\end{array}$ & $\begin{array}{l}\text { Persistent AKI } \\
N=483\end{array}$ \\
\hline \multicolumn{4}{|l|}{ KDIGO stage } \\
\hline Stage $1 n(\%)$ & $464(45.8)$ & $372(70.3)$ & $92(19.0)$ \\
\hline Stage $2 n(\%)$ & $177(17.5)$ & $76(14.4)$ & $101(20.9)$ \\
\hline Stage $3 n(\%)$ & $371(36.7)$ & $81(15.3)$ & $290(60.0)$ \\
\hline RRT & 220 & 0 & 220 \\
\hline \multicolumn{4}{|l|}{ Causes of RRT } \\
\hline Oliguria/anuria $n(\%)$ & $179(81.4)$ & 0 & $179(81.4)$ \\
\hline Severe acidosis $(\mathrm{pH}<7.15) n(\%)$ & 79 (35.9) & 0 & $79(35.9)$ \\
\hline Refractory hyperkalemia $\left(\mathrm{K}^{+}>6 \mathrm{mmol} / \mathrm{L}\right) n(\%)$ & $64(29.1)$ & 0 & $64(29.1)$ \\
\hline Fluid overload with organ edema $n(\%)$ & $20(9.1)$ & 0 & $20(9.1)$ \\
\hline SIRS with MODS $n(\%)$ & $15(6.8)$ & 0 & $15(6.8)$ \\
\hline Others ${ }^{a} n(\%)$ & $15(6.8)$ & 0 & $15(6.8)$ \\
\hline Weaning from RRT $n(\%)$ & $71(32.3)$ & 0 & $71(32.3)$ \\
\hline LOS in ICU (days) & $7(4-15)$ & $6(3-11)$ & $9(5-17)$ \\
\hline LOS in hospital (days) & $18(10-27)$ & $18(12-27)$ & $17(8-28)$ \\
\hline 28-day mortality $n(\%)$ & $297(29.3)$ & $95(18.0)$ & $202(41.8)$ \\
\hline
\end{tabular}

Continuous variables are presented as median and interquartile range

$A K I$ acute kidney injury, KDIGO kidney disease improving global outcomes, RRT renal replacement therapy, LOS length of stay, SIRS systemic inflammatory response syndrome, MODS multiple organ dysfunction

${ }^{a}$ signs and symptoms of uremia (bleeding, pericarditis and encephalopathy), azotemia (BUN $>100 \mathrm{mg} / \mathrm{dL}$ ), hypernatremia, high urea/creatinine levels 


\section{Discussion}

The detection rate of AKI based on serum creatinine change depends on the frequency of serum testing. However, some studies did not record the changes of creatinine before patients were transferred to ICU, which makes it impossible to accurately calculate the duration of kidney injury for some patients who already had AKI at admission. Patients with AKI before ICU often have serious illness condition, more complications and higher mortality. Therefore, in order to reduce the excessive confounding factors, we excluded the patients who developed AKI before admitted to ICU. Moreover, we excluded the patients diagnosed as AKI within the first $6 \mathrm{~h}$ after ICU admission, considering that the kidney injury at this time may actually occurred before ICU. We defined AKI that appeared in the first $72 \mathrm{~h}$ after ICU admission as new-onset AKI because the previous studies found that approximately $93.1 \%$ of AKI occurred in the first $72 \mathrm{~h}$ after admitted to ICU in critically ill patients [5-7]. The definition of new-onset AKI in our study might prevent the influence of duration of AKI before ICU admission, which facilitated the precise grouping of transient AKI and persistent AKI. As we know, this study represented the largest and the most extensive analysis of new-onset AKI among ICU adults in China.

We calculated the incidence of new-onset AKI at $30.0 \%$ among 3374 patients from 17 ICUs of 16 regional central hospitals encompassing a wide range of clinical settings. Our estimate was lower than the $57.3 \%$ reported in EPI-AKI study [2], mainly because this study excluded patients with AKI before ICU admission and patients with CKD. We also assessed the incidence of new-onset AKI at $42.8 \%$ among septic patients in our study. The prevalence of AKI in patients with severe sepsis or septic shock has been reported to be in the range of $30-40 \%$ during the last two decades [21, 22], while the recent FINNAKI study showed $53 \%$ of 918 patients with severe sepsis met the KDIGO criteria for AKI [23]. The main reason for the higher prevalence was that recent consensus definitions of AKI had increased the sensitivity of its detection. The prevalence of new-onset AKI among septic patients in our study was lower than FINNAKI study, this difference may represent the patients who have already had AKI before ICU admission. Logistic regression in our study showed that coronary heart disease, hypertension, chronic liver disease, use of nephrotoxic drugs, sepsis, SOFA score, APACHEII score and use of vasopressors were independent risk factors for new-onset AKI. These findings were consistent with previous findings [24, 25]. Sepsis was the most common contributing factor to new-onset AKI.

The initiation of RRT among new-onset AKI patients reviewed the defects of traditional ways of treatment in
China. Three leading reasons to start RRT were similar to the results of B.E.S.T. Kidney study [26], which showed the most common initiation of RRT was oliguria/ anuria, followed by high urea/creatinine, metabolic acidosis and fluid overload. However, only a few patients in our study started RRT because of high urea/ creatinine, this may reflect Chinese doctors' insensitivity to high urea/creatinine level. In addition, systemic inflammatory response syndrome (SIRS) with multiple organ dysfunction syndrome (MODS) was the initiation of RRT in some AKI patients, its proportion (6.8\%) is significantly higher than that of other countries although it might be the co-reason for RRT initiation [26]. This may be because some intensivists still believe that RRT is a remedy to reduce systemic inflammation.

Our study found that the new-onset AKI was associated with higher 28-day mortality and longer length of hospital stay. The patients with new-onset AKI had a probability for 28-day mortality that was 0.6 times higher compared with those without AKI, even after adjusting for a large number of possible confounders. However, only persistent AKI is associated with increased 28-day mortality rather than transient AKI. The prognostic impact of transient AKI remains controversy in previous studies [8,10-12]. A large multicenter cohort study [11] demonstrated that both transient and persistent AKI were independently associated with a poor outcome, and that patients with persistent AKI had a higher risk of death than patients with transient AKI. Our results differ somewhat from previous studies [11, 12 ], which focused on all hospitalized patients. Our results are similar to a recent study of septic patients that also came to the conclusion that only persistent AKI is associated with significantly higher 28-day mortality [27]. The strong association between persistent AKI and the poor outcome can be explained as follows: the long duration of AKI indicates the exacerbated physiologic profiles and the poor prognosis. In addition, the pathophysiological mechanism of transient AKI and persistent AKI may be different. Transient AKI is considered to be "prerenal dysfunction", while AKI that has not been recovered for a long time is more related to "acute tubular necrosis" [28]. Our results suggest for future clinical research to consider that quick and spontaneous resolving AKI may dilute trials and should not be the target of new treatment trials, and highlight the importance for clinician to put more value on duration of kidney injury. The uncertainty of illness makes it difficult for intensivists to predict transient and persistent AKI, but the several independent variables reported in Table 3 could help to distinguish persistent AKI. In our result, none of the transient AKI patient had RRT even though $15.3 \%$ of them had stage 3 AKI (Table 4), and in clinical practice, RRT should be carefully considered to avoid 
unnecessary intervention for patients who are unlikely to develop persistent AKI.

The strengths of this study are the large sample size, the use of a consensus definition for AKI diagnosis, and actively searching for baseline serum creatinine, being available in the majority of the included patients, despite the high proportion of emergency admissions. On the other hand, this study also has limitations. First, patients with CKD were excluded. The prognosis of CKD is different from AKI with a normal baseline creatinine [29]. Furthermore, a patient with CKD has a high, abnormal and unstable creatinine level, the $0.3 \mathrm{mg} / \mathrm{dl}$ change cannot accurately reflect the development of AKI, and it may show an unimportant change of GFR. However, excluding CKD patients did make our results of AKI incidence lower than others. Second, as a retrospective analysis based on database of a prospective cohort study, we did not control the treatment of AKI, for AKI duration could be changed depending on the treatment. Third, our definition of persistent AKI was that kidney injury beyond $48 \mathrm{~h}$ from AKI onset, which was shorter than some previous studies [12, $30]$, this may reduce the severity of persistent AKI patients and influenced the 28-day mortality.

\section{Conclusions}

In general ICU patients, $30.0 \%$ of the patients developed new-onset AKI. The risk factors associated with newonset AKI included coronary heart disease, hypertension, chronic liver disease, use of nephrotoxic drugs, sepsis, SOFA score, APACHEII score and use of vasopressors. The new-onset AKI was an independent risk factor for 28-day mortality. Transient AKI is not associated with 28-day death, while persistent AKI is strongly associated with worse outcome.

\section{Abbreviations}

AKI: Acute kidney injury; ICU: Intensive care unit; SOFA: Sequential organ failure assessment; APACHEIl: Acute physiology and chronic health evaluationll; RRT: Received renal replacement therapy; HR: Hazard ratio; Cl: Confidence interval; P: Probability; RIFLE: Risk, injury, failure, loss of kidney function, and end-stage renal disease; AKIN: Acute kidney injury network; KDIGO: Kidney disease improving global outcomes; CCCST: China Critical Care Sepsis Trial; eGFR: Estimated glomerular filtration rate; MDRD: Modification of Diet in Renal Disease; SD: Standard deviation; OR: Odds ratio; SIRS: Systemic inflammatory response syndrome; MODS: Multiple organ dysfunction syndrome
}

\section{Supplementary Information}

The online version contains supplementary material available at https://doi. org/10.1186/s12882-021-02503-x.

Additional file 1 : Table S1. Baseline characteristics of new-onset AKI patients stratified by duration of acute kidney injury. Table $\mathbf{S 2}$.
Characteristics and rates of new-onset AKI and 28-day death for each individual center.

\section{Acknowledgements}

We thank the 17 collaborating centers of the China Critical Care Sepsis Trial (CCCST) workgroup: Department of Critical Care Medicine, Fuxing Hospital, Capital Medical University, Beijing, China; Department of Critical Care Medicine, West China Hospital, Sichuan University, Sichuan, China; Medical Intensive Care Unit, Peking Union Medical College Hospital, Beijing, China; Department of Critical Care Medicine, Guangdong Geriatric Institute, Guangdong General Hospital, Guangdong, China; Department of Critical Care Medicine, The First Affiliated Hospital of China Medical University, Shenyang, China; Surgical Intensive Care Unit, Department of Anaesthesiology,

ZhongShan Hospital, FuDan University, Shanghai, China; Intensive Care Unit, The First Hospital of Jilin University, Changchun, China; Department of Critical Care Medicine, China-Japan Friendship Hospital, Beijing, China; Department of Critical Care Medicine, Beijing Friendship Hospital, Capital Medical University, Beijing China; Surgical Intensive Care Unit, Beijing Chaoyang Hospital, Capital Medical University, Beijing, China; Department of Respiratory and Critical Care Medicine, Beijing Institute of Respiratory Medicine, Beijing Chaoyang Hospital, Capital Medical University, Beijing, China; Department of Critical Care Medicine, General Hospital of Ningxia Medical University, Ningxia, China; Department of Critical Care Medicine, Xiangya Hospital, Central South University, Changsha, China; Department of Critical Care Medicine, Beijing Tongren Hospital, Capital Medical University, Beijing, China; Department of Critical Care Medicine, Peking University Third Hospital, Beijing, China; Surgical Intensive Care Unit, Xuanwu Hospital, Capital Medical University, Beijing, China; Department of Critical Care Medicine, Beijing Tiantan Hospital, Capital Medical University, Beijing, China.

\section{Authors' contributions}

YYJ contributed to data interpretation, statistical analysis, drafting of the manuscript and critical revision of the manuscript. XXM, HMJ, XZ, MPW, WL contributed to data collection and data interpretation. WXL conceived and designed the study, performed critical revision of the manuscript. All authors commented on previous versions of the manuscript. All authors read and approved the final manuscript.

\section{Fundings}

This study was supported by grants from the Beijing Municipal Science \& Technology Commission (no. Z191100006619032) and National Science and Technology Support Program (no. 2012BAl11B).

Availability of data and materials

The datasets used and/or analyzed during the current study are available from the corresponding author on reasonable request.

\section{Declarations}

\section{Ethics approval and consent to participate}

The study was approved in all participating ICUs by their Hospital Human Ethics Committee. The chief ethics number was 2013FXHEC-KY2018. The registration number was ChiCTR-ECH-13003934. The study followed the ethical principles of the Declaration of Helsinki 1964. Informed consent from patients or their next of kin was obtained before patients joined in the study.

\section{Consent for publication}

The manuscript does not contain any individual person's data in any form.

Competing interests

The authors declare that they have no competing interests.

\section{Author details}

'Department of Surgical Intensive Critical Unit, Beijing Chao-yang Hospital, Capital Medical University, 8 Gongren Tiyuchang Nanlu, Chaoyang District, 100020 Beijing, China. ${ }^{2}$ Department of Critical Care Medicine, Fuxing Hospital, Capital Medical University, Beijing, China. ${ }^{3}$ Department of Epidemiology and Health Statistics, School of Public Health, Capital Medical University, Beijing, China. 
Received: 21 February 2021 Accepted: 14 August 2021

Published online: 25 August 2021

\section{References}

1. Ronco C, Bellomo R, Kellum JA. Acute kidney injury. Lancet. 2019; 394:194964.

2. Hoste EA, Bagshaw SM, Bellomo R, Cely CM, Colman R, Cruz DN, et al. Epidemiology of acute kidney injury in critically ill patients: the multinational AKI-EPI study. Intensive Care Med. 2015; 41:1411-23.

3. Chawla LS, Eggers PW, Star RA, Kimmel PL. Acute kidney injury and chronic kidney disease as interconnected syndromes. N Engl J Med. 2014; 371:5866.

4. Khwaja A. KDIGO clinical practice guidelines for acute kidney injury. Nephron Clin Pract. 2012; 120:179-84.

5. Koyner JL, Carey KA, Edelson DP, Churpek MM. The development of a machine learning inpatient acute kidney injury prediction model. Crit Care Med. 2018; 46: 1070-7.

6. Honore PM, Jacobs R, Joannes-Boyau O, De Regt J, Boer W, De Waele E, et al. Septic AKI in ICU patients. Diagnosis, pathophysiology, and treatment type, dosing, and timing: a comprehensive review of recent and future developments. Ann Intensive Care. 2011; 1:32.

7. Vaara ST, Pettilä V, Kaukonen KM, Bendel S, Korhonen AM, Bellomo R, et al. The attributable mortality of acute kidney injury: a sequentially matched analysis. Crit Care Med. 2014; 42:878-85.

8. Han SS, Kim S, Ahn SY, Lee J, Na KY. Duration of acute kidney injury and mortality in critically ill patients: a retrospective observational study. BMC Nephrol. 2013; 14:133.

9. Mehta S, Chauhan K, Patel A, Patel S, Pinotti R, Nadkarni GN, et al. The prognostic importance of duration of AKI: a systematic review and metaanalysis. BMC Nephrol.2018; 19:91.

10. Coca SG, King JT, Rosenthal RA, Perkal MF, Parikh CR. The duration of postoperative acute kidney injury is an additional parameter predicting long-term survival in diabetic veterans. Kidney Int. 2010; 78:926-33.

11. Uchino S, Bellomo R, Bagshaw SM, Goldsmith D. Transient azotaemia is associated with a high risk of death in hospitalized patients. Nephrol Dial Transplant. 2010; 25:1833-9.

12. Brown JR, Kramer RS, Coca SG, Parikh CR. Duration of acute kidney injury impacts long-term survival after cardiac surgery. Ann Thorac Surg. 2010; 90: $1142-8$

13. Gammelager $H$, Christiansen $C F$, Johansen MB, Tonnesen $E$, Jespersen $B$, Sorensen HT. Three-year risk of cardiovascular disease among intensive care patients with acute kidney injury: a population-based cohort study. Crit Care. 2014; 18:492

14. Palevsky PM, Liu KD, Brophy PD, Chawla LS, Parikh CR, Thakar CV, et al. KDOQI US commentary on the $2012 \mathrm{KDIGO}$ clinical practice guideline for acute kidney injury. Am J Kidney Dis. 2013; 61:649-72.

15. Bellomo R, Ronco C, Kellum JA, Mehta RL, Palevsky P. Acute Dialysis Quality Initiative workgroup. Acute renal failure-definition, outcome measures, animal models, fluid therapy and information technology needs: the Second International Consensus Conference of the Acute Dialysis Quality Initiative (ADQI) Group. Crit Care. 2004;8:R204-12

16. Pilarczyk K, Edayadiyil-Dudasova M, Wendt D, Demircioglu E, Benedik J, Dohle DS, et al. Urinary [TIMP-2]*[IGFBP7] for early prediction of acute kidney injury after coronary artery bypass surgery. Ann Intensive Care. 2015; 5:50.

17. Kashani K, Al-Khafaji A, Ardiles T, Artigas A, Bagshaw SM, Bell M, et al. Discovery and validation of cell cycle arrest biomarkers in human acute kidney injury. Crit Care. 2013; 17:R25.

18. Singer M, Deutschman CS, Seymour CW, Shankar-Hari M, Annane D, Bauer $M$, et al. The third international consensus definitions for sepsis and septic shock (Sepsis-3). JAMA. 2016; 315:801-10.

19. Chawla LS, Bellomo R, Bihorac A, Goldstein SL, Siew ED, et al. Acute kidney disease and renal recovery: consensus report of the Acute Disease Quality Initiative (ADQI) 16 Workgroup. Nat Rev Nephrol. 2017; 13:241-57.

20. Kellum JA, Sileanu FE, Bihorac A,Hoste EAJ, Chawla LS. Recovery after acute kidney injury. Am J Resp Crit Care. 2017; 195:784-91.

21. Bagshaw SM, George C, Bellomo R. Early acute kidney injury and sepsis: a multicentre evaluation. Crit Care. 2008; 12:R47.

22. Oppert M, Engel C, Brunkhorst FM, Bogatsch $H$, Reinhart $K$, Frei $U$, et al. Acute renal failure in patients with severe sepsis and septic shock - a significant independent risk factor for mortality: results from the German Prevalence Study. Nephrol Dial Transplant. 2008; 23:904-9.

23. Poukkanen M, Vaara ST, Pettila V, Kaukonen KM, Korhonen AM, Hovilehto S, et al. Acute kidney injury in patients with severe sepsis in Finnish intensive care units. Acta Anaesthesiol Scand. 2013; 57:863-72.

24. Mehta RL, Pascual MT, Gruta CG, Zhuang SP, Chertow GM. Refining predictive models in critically ill patients with acute renal failure. J Am Soc Nephrol. 2002; 13:1350-7.

25. Uchino S, Kellum JA, Bellomo R, Doig GS, Ronco C. Acute renal failure in critically ill patients: a multinational, multicenter study. JAMA. 2005; 294:813-

26. Uchino S, Bellomo R, Morimatsu H, Morgera S, Schetz M, Tan I, et al. Continuous renal replacement therapy: a worldwide practice survey. The beginning and ending supportive therapy for the kidney (B.E.S.T. kidney) Investigators. Intensive Care Med. 2007; 33:1563-70.

27. Uhel F, PetersSengers H, Falahi F, Scicluna BP, van Vught LA, Bonten MJ, et al. Mortality and host response aberrations associated with transient and persistent acute kidney injury incritically ill patients with sepsis: a prospective cohort study. Intensive Care Med. 2020;46:1576-89.

28. Lameire N, Van Biesen W, Vanholder R: Acute renal failure. Lancet. 2005; 365: 417-30.

29. Abdalrahim MS, Khalil AA, Alramly M, Alshlool KN, Abed MA, Moser DK. Preexisting chronic kidney disease and acute kidney injury among critically ill patients. Heart Lung. 2020; 49:626-9.

30. Federspiel CK, Itenov TS, Mehta K, Hsu RK, Bestle MH. Duration of acute kidney injury in critically ill patients. Ann Intensive Care. 2018; 8:30.

\section{Publisher's Note}

Springer Nature remains neutral with regard to jurisdictional claims in published maps and institutional affiliations.

Ready to submit your research? Choose BMC and benefit from:

- fast, convenient online submission

- thorough peer review by experienced researchers in your field

- rapid publication on acceptance

- support for research data, including large and complex data types

- gold Open Access which fosters wider collaboration and increased citations

- maximum visibility for your research: over $100 \mathrm{M}$ website views per year

At $\mathrm{BMC}$, research is always in progress.

Learn more biomedcentral.com/submissions 